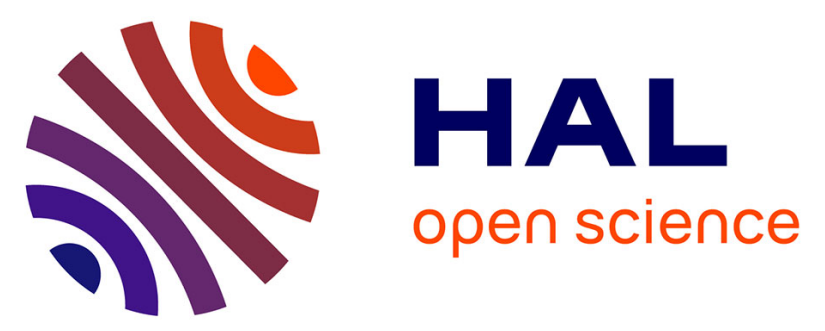

\title{
Concevoir des environnements virtuels éducatifs avec les utilisateurs finaux : Exemple du projet VirtualiTeach
}

Emilie Loup-Escande, Eric Jamet, Martin Ragot, Séverine Erhel, Nicolas Michinov, Clément Peltier, Thomas Lopez

\section{- To cite this version:}

Emilie Loup-Escande, Eric Jamet, Martin Ragot, Séverine Erhel, Nicolas Michinov, et al.. Concevoir des environnements virtuels éducatifs avec les utilisateurs finaux : Exemple du projet VirtualiTeach. Terminal. Technologie de l'information, culture \& société, 2015, 117, pp.1-19. 10.4000/terminal.1093 . hal-01784177

\section{HAL Id: hal-01784177 \\ https://hal.univ-rennes2.fr/hal-01784177}

Submitted on 3 May 2018

HAL is a multi-disciplinary open access archive for the deposit and dissemination of scientific research documents, whether they are published or not. The documents may come from teaching and research institutions in France or abroad, or from public or private research centers.
L'archive ouverte pluridisciplinaire HAL, est destinée au dépôt et à la diffusion de documents scientifiques de niveau recherche, publiés ou non, émanant des établissements d'enseignement et de recherche français ou étrangers, des laboratoires publics ou privés. 


\section{Terminal}

Technologie de l'information, culture \& société

$117 \mid 2015$

Pratique des outils numériques

\section{Concevoir des environnements virtuels éducatifs avec les utilisateurs finaux : Exemple du projet VirtualiTeach}

Designing virtual environments with end users: The case of the VirtualiTeach project

Émilie Loup-Escande, Éric Jamet, Martin Ragot, Séverine Erhel, Nicolas Michinov, Clément Peltier et Thomas Lopez

\section{(2) OpenEdition}

\section{Journals}

Édition électronique

URL : http://journals.openedition.org/terminal/1093

DOI : 10.4000/terminal. 1093

ISSN : 2429-4578

Éditeur

CREIS-Terminal

Ce document vous est offert par Université Rennes 2

Référence électronique

Émilie Loup-Escande, Éric Jamet, Martin Ragot, Séverine Erhel, Nicolas Michinov, Clément Peltier et Thomas Lopez, «Concevoir des environnements virtuels éducatifs avec les utilisateurs finaux: Exemple du projet VirtualiTeach », Terminal [En ligne], 117 | 2015, mis en ligne le 25 novembre 2015, consulté le 03 mai 2018. URL : http://journals.openedition.org/terminal/1093 ; DOI : 10.4000/ terminal.1093

Ce document a été généré automatiquement le 3 mai 2018.

tous droits réservés 


\title{
Concevoir des environnements virtuels éducatifs avec les utilisateurs finaux : Exemple du projet VirtualiTeach
}

\author{
Designing virtual environments with end users: The case of the VirtualiTeach \\ project
}

Émilie Loup-Escande, Éric Jamet, Martin Ragot, Séverine Erhel, Nicolas Michinov, Clément Peltier et Thomas Lopez

Nous remercions les chefs de travaux, les enseignants et les élèves qui ont participé activement aux différentes actions menées, ainsi que les partenaires industriels du projet (CLARTE, CEA List, CADWARE, Académie de Créteil, Académie de Nantes, Académie de Rennes) et les financeurs (Caisse des Dépôts et des Consignations dans le cadre des Investissements d'Avenir, la région Pays de la Loire et la région Bretagne).

\section{Introduction}

Depuis quelques années, des Technologies de l'Information et de la Communication pour l'Enseignement (TICE) mobilisant les technologies de la réalité virtuelle, également connues sous le terme d'Environnements Virtuels Éducatifs (EVE), sont développées (Mikropoulos, Natsis, 2011; Plantard, 1999). Un EVE peut être défini comme un environnement virtuel - fondé sur un modèle pédagogique spécifique en fonction d'objectifs didactiques - qui fournit aux utilisateurs des expériences qu'ils ne peuvent pas trouver (totalement ou partiellement) dans le monde réel et qui favorise des apprentissages chez les apprenants (Mikropoulos, Natsis, op. cit.). Ils font l'objet de nombreuses recherches sur les technologies utilisées (Dede, 2009), les disciplines ciblées (Ausburn, Ausburn, 2004), les niveaux scolaires concernés (Hew, Cheung, 2010) ainsi que 
sur les modèles et les critères à considérer pour la conception et l'évaluation de telles ressources pédagogiques (Freitas, Neumann, 2009). Si les TICE mobilisant la réalité virtuelle peuvent être des ressources pédagogiques complémentaires à des outils plus traditionnels comme les manuels scolaires (Codd, Choudhury, 2011; Virvou, Katsionis, Manos, 2005), de nombreuses variables peuvent compromettre leur efficacité. L'utilisabilité et la correspondance avec les besoins des « usagers ", qu'il s'agisse d'acteurs institutionnels (Crétin-Pirolli, Cottier, 2009) ou d'utilisateurs finaux (typiquement, les enseignants et les élèves), font partie de ces variables.

2 Pour assister les concepteurs en ce qui concerne l'inclusion de ces utilisateurs finaux dans le processus de conception, la norme ISO 9241-210 (2010) a formalisé une démarche de la conception centrée-utilisateur permettant ainsi de rendre les systèmes interactifs (par exemple, les TICE mobilisant la réalité virtuelle) plus utiles, utilisables et acceptables. $\mathrm{Si}$ cette démarche et les méthodes qu'elle emploie sont souvent utilisées pour la conception de dispositifs interactifs traditionnels (Fleury, Jamet, Loup-Escande et al., 2013 ; Jamet, Trémenbert, Deguillard et al., 2011), son utilisation est moins fréquente en ce qui concerne les ressources issues des technologies de la réalité virtuelle. De plus, les travaux suggérant l'utilisation de la conception centrée-utilisateur en réalité virtuelle (Hix, Swan, Gabbard et al., 1999) n'impliquent pas nécessairement des utilisateurs finaux mais plutôt des sujets tout venant non représentatifs des utilisateurs finaux réels (Ganier, Hoareau, Devillers, 2013). Ces travaux portent généralement sur l'utilisabilité et la tâche elle-même est souvent éloignée de la tâche réelle.

3 Dans le domaine éducatif, les utilisateurs finaux des TICE seront les personnes qui les utiliseront dans le cadre de leur activité professionnelle (par exemple, les enseignants) ou scolaire (par exemple, les élèves). Si la conception des technologies d'apprentissage et plus spécifiquement d'EVE fait l'objet de quelques travaux (Burkhardt, Lourdeaux, Melletd'Huart, 2003), cela reste un domaine dans lequel beaucoup de questions tant sur le plan théorique que méthodologique restent à investiguer.

4 L'objectif de notre article est de formaliser ce qu'est une conception avec et pour les utilisateurs finaux (c'est-à-dire, chefs de travaux ${ }^{1}$, enseignants et élèves) dans le contexte des EVE visant à favoriser l'apprentissage de concepts scientifiques et à en identifier les apports. Pour ce faire, nous analysons la démarche de conception centrée-utilisateur que nous avons instaurée dans le cadre du projet VirtualiTeach ${ }^{2}$. Ce projet a pour ambition de concevoir des EVE pour l'apprentissage de concepts scientifiques dans des filières technologiques et professionnelles. Les technologies émergentes ont été envisagées comme un moyen favorisant une approche inductive selon laquelle la pratique permettait à l'élève de mettre en évidence des notions théoriques ensuite formalisées avec les enseignants. Si ces filières sont souvent équipées de dispositifs réels, ces derniers peuvent paraitre insuffisants lorsqu'il s'agit d'observer des phénomènes non visibles à l'œil nu. Ces phénomènes deviennent alors perceptibles grâce à la réalité virtuelle qui permet de rendre ces phénomènes observables au travers des modélisations et des animations.

Dans la suite de l'article, nous fournissons des éléments de définition concernant la conception centrée-utilisateur. La section 3 détaille la manière dont nous avons mis en œuvre cette démarche au travers des méthodes et des résultats observés pour spécifier les contextes d'utilisation et les besoins des usagers ainsi que pour comprendre l'utilisation de ces outils dans des situations pédagogiques réelles. En conclusion, nous synthétisons la contribution des différents usagers, les apports de la conception centrée- 
utilisateur et les précautions à considérer quant à l'implication des chefs de travaux, des enseignants et des élèves dans la conception centrée-utilisateur de ces TICE émergentes.

\section{Conception centrée-utilisateur}

6 Selon la norme ISO 9241-210 de 2010, la démarche de conception centrée-utilisateur est caractérisée par six principes: 1) la conception est fondée sur une compréhension explicite des utilisateurs, des tâches et des environnements, 2) les utilisateurs sont impliqués dans la conception et le développement, 3) la conception est orientée et dirigée par l'évaluation centrée sur l'utilisateur, 4) le processus est itératif jusqu'à obtention du résultat souhaité, 5) la conception couvre l'expérience de l'utilisateur dans son intégralité, 6) l'équipe de conception inclut des compétences pluridisciplinaires.

7 L'application de ces principes implique l'évaluation de plusieurs dimensions ergonomiques comme l'utilité, l'utilisabilité, l'acceptabilité, l'expérience-utilisateur et l'immersion. L'utilité fait référence à l'adéquation entre les fonctionnalités de l'application d'une part, et les besoins, les buts des utilisateurs et les contextes d'usages de l'application d'autre part (Loup-Escande, Burkhardt, Richir, 2013). D'après la norme ISO 9241-11, l'utilisabilité correspond au degré selon lequel un produit peut être utilisé avec efficacité, efficience et satisfaction, par des utilisateurs identifiés, pour atteindre des buts définis, dans un contexte d'utilisation spécifié. L'acceptabilité fait référence aux représentations a priori des utilisateurs sur une technologie, par exemple en termes d'utilité, d'utilisabilité perçue ou d'intention d'usage (Venkatesh, Morris, Davis et al., 2003). L'expérience-utilisateur résulte de l'expérience subjective produite suite à l'interaction entre un utilisateur et un dispositif informatique (Hassenzahl, 2011). L'immersion correspond au degré et à la qualité avec lesquels l'interface du système contrôle les entrées sensorielles pour chaque modalité de perception et d'action (Witmer, Singer, 1998). Ces dimensions sont particulièrement intéressantes à investiguer dans le domaine des TICE mobilisant la réalité virtuelle dans la mesure où ces dernières reposent sur des dispositifs émergents, de nouveaux modes d'interaction, de nouvelles pratiques d'enseignement et de nouveaux usages pédagogiques.

8 La démarche de conception centrée-utilisateur implique la réalisation de quatre tâches. La première tâche consiste à comprendre et à spécifier le contexte d'utilisation par une analyse de l'environnement dans lequel l'outil sera intégré. La seconde tâche consiste à analyser les exigences des utilisateurs. La troisième tâche est relative à la production de solutions de conception (par exemple, preuves de concept, prototypes) sur la base de connaissances techniques d'une part, de connaissances sur les utilisateurs et le contexte d'utilisation d'autre part (Tableau 1). Enfin, la quatrième tâche concerne l'évaluation de ces solutions.

Tableau 1. Systèmes développés (colonne de droite) et catégories de solutions de conception (colonne de gauche)

\begin{tabular}{|l|l|}
\hline Systèmes développés & Catégories de solutions de conception \\
\hline Écoulement de fluide & Preuve de concept \\
\hline Confort acoustique & Preuve de concept \\
\hline
\end{tabular}




\begin{tabular}{|l|l|}
\hline Moteur thermique & Preuve de concept \\
\hline Schéma cinématique & Preuve de concept \\
\hline Gamme de montage & Preuve de concept \\
\hline Projection d'enduit & Preuve de concept \\
\hline Vertige & Prototype \\
\hline Éditeur cinématique & Prototype \\
\hline Virtualkart & Prototype / Application finale \\
\hline Venturi & Prototype / Application finale \\
\hline Forces de frottements statiques & Prototype / Application finale \\
\hline Résistance des matériaux & Prototype / Application finale \\
\hline
\end{tabular}

9 Dans le cadre du projet, nous avons réalisé chacune de ces tâches au travers d'actions spécifiques impliquant des utilisateurs finaux variés et de méthodes utilisées en ergonomie (Maguire, 2001). Les méthodes utilisées dans le cadre du projet ont été choisies pour des raisons de complémentarité, dans la mesure où elles visaient à produire des données de nature différente au cours de la conception. Cette différence concerne deux aspects. Le premier concerne le niveau de la subjectivité des données : par exemple, les entretiens semi-directifs permettent de recueillir des données subjectives telles que les représentations tandis que les tests utilisateurs permettent également d'accéder à des données objectives issues de la confrontation des utilisateurs aux EVE. Le second aspect fait référence à la nature même des données : par exemple, les données des entretiens proviennent des représentations que les utilisateurs ont eu à l'issue de l'utilisation des EVE dans des contextes existants, les méthodes prospectives telles que les brainstormings favorisent l'évocation d'idées et d'usages fondés sur la projection des utilisateurs dans une situation future probable, les évaluations heuristiques ne sont basées que sur les représentations et l'expertise des concepteurs.

Les actions ont été mises en œuvre selon le séquencement et l'échelle temporelle décrits dans la Figure 1. 
Fig. 1. Phases du processus de conception et actions réalisées au cours du projet

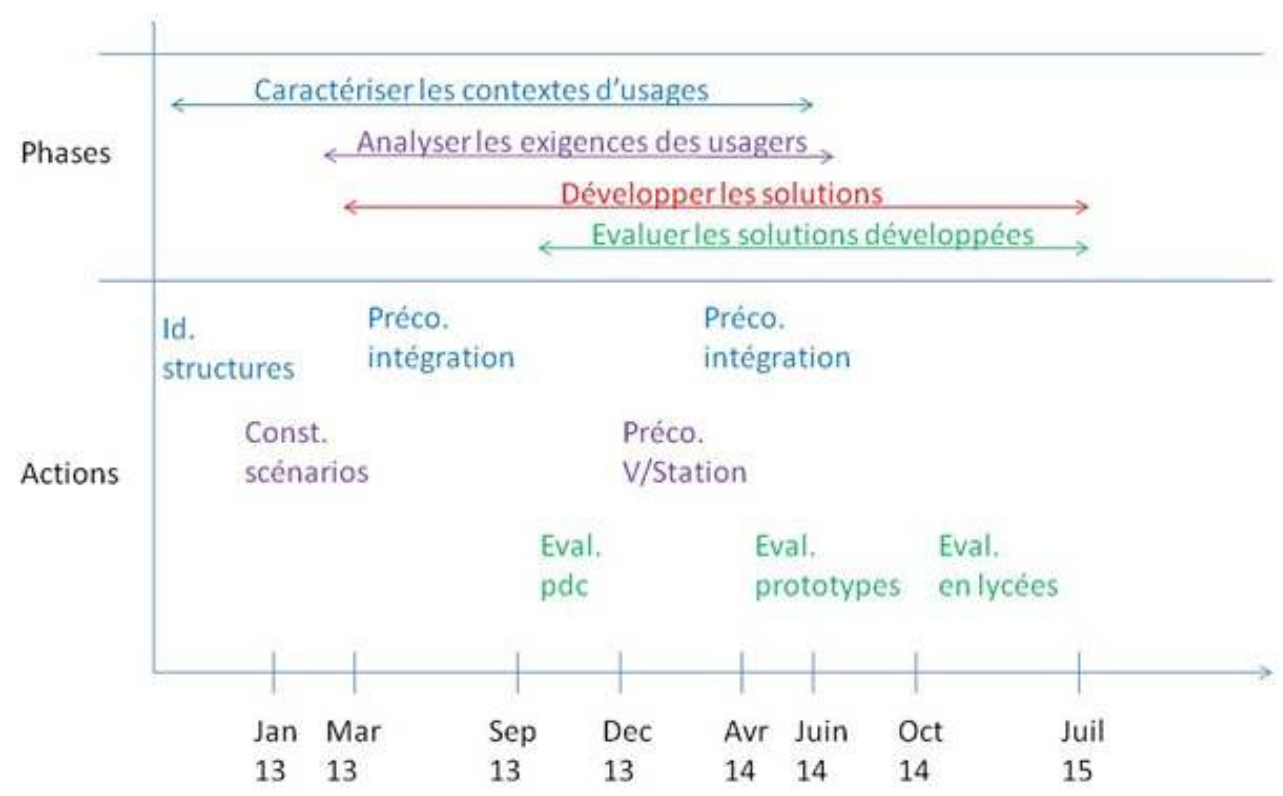

Légende :

Id. structures : identifier les structures utilisatrices

Préco. Intégration : préconisations sur l'intégration physique et pédagogique de la plateforme

Const. Scénarios : co-construction de scénarios pédagogiques

Préco. V/Station : recommandations pour l'ergonomie du dispositif matériel de réalité virtuelle

Eval. pdc : évaluation des preuves de concepts

Eval. prototypes : évaluation de prototypes

Eval. en lycées : évaluation en lycées

\section{Actions réalisées, méthodes utilisées et données collectées auprès des chefs de travaux, des enseignants et des élèves}

\section{Caractériser les contextes d'usages}

Identification des structures utilisatrices

11 La mise en place d'une démarche de conception centrée-utilisateur dans un projet d'innovation pédagogique nécessite la constitution d'un consortium composé non seulement d'organismes en charge du développement des TICE émergentes, mais aussi de partenaires usagers de ces technologies (c'est-à-dire, formations cibles, lycées partenaires).

Pour caractériser les contextes d'usage, nous avons d'abord identifié les structures utilisatrices au travers de réunions avec des acteurs institutionnels, à savoir les représentants des trois inspections académiques (Rennes, Nantes et Créteil) et du Ministère de l'Éducation Nationale. Ces réunions ont ainsi permis d'isoler les formations concernées par le projet et de préciser les filières techniques et professionnelles concernées. Ainsi, il a été conclu que les ressources développées dans le cadre du projet VirtualiTeach seraient intéressantes pour des enseignants et des élèves du baccalauréat technologique STI2D , aussi bien dans le Tronc Commun que dans les spécialités «Énergie 
et Environnement ", "Architecture et Construction » et "Innovation technologique et Éco-conception ", ainsi que pour des enseignants et des élèves de plusieurs baccalauréats professionnels (c'est-à-dire, Électrotechnique, Conception Mécanique, Construction) et de différents BTS (c'est-à-dire, Conception de Produits Industriels, Conception et Réalisation de Systèmes Automatiques, Génie Civil, Industrialisation des Produits Mécaniques). Ces choix de filières ont été faits en regard de la pertinence avérée dans la littérature de la réalité virtuelle pour l'acquisition de connaissances scientifiques et techniques [par exemple, 33] intrinsèques à ces formations. Par ailleurs, les lycées partenaires dont les chefs de travaux, les enseignants et les élèves pourraient être impliqués dans la conception des futures TICE pour l'Académie de Nantes ${ }^{4}$, de l'Académie de Créteil ${ }^{5}$ et de l'Académie de Rennes ${ }^{6}$ ont été identifiés, notamment sur la base de leur proximité géographique avec les partenaires du projet. Les acteurs institutionnels impliqués à cette étape du processus ont suivi l'avancement du projet au travers des réunions annuelles organisées par le porteur.

\section{Préconisations sur l'intégration physique et pédagogique de la plate-forme}

13 Si de plus en plus de TICE mobilisant la réalité virtuelle sont implémentées, leur intégration dans des situations pédagogiques réelles implique une réorganisation des espaces de travail (c'est-à-dire, les équipements peuvent être imposants) et des pratiques pédagogiques (c'est-à-dire, comment intégrer la réalité virtuelle dans une séance de travaux pratiques ?). Pour établir des préconisations, nous avons réalisé une revue de la littérature pour s'inspirer des travaux antérieurs sur les modèles d'organisation des salles de classes équipées de ressources informatiques et sur l'utilisation de la réalité virtuelle dans le domaine éducatif, ainsi que des focus group avec les acteurs directement concernés pour favoriser la production d'idées innovantes (Bruseberg, McDonagh-Philip, 2002). Un focus group est une activité de groupe ayant pour objet de se «focaliser » sur des aspects précis d'un objet d'étude. Cette méthode consiste à créer des petits groupes d'utilisateurs faisant partie de la population cible du produit que l'on fait interagir autour d'un sujet pertinent pour l'étude à réaliser. En sortie, sont généralement recueillies des opinions, attentes, motivations et attitudes, à partir des usages, pratiques, expériences vécues des participants.

14 Nous avons réalisé un état de l'art sur les modèles d'organisation des salles de classe qui a montré que l'organisation en îlots (Baker, 1984; Whiteside, Brooks, Walker, 2010) facilitait la pédagogie active et collaborative (Fisher, 2010), la collaboration entre les élèves et les interactions entre les élèves et l'enseignant, ainsi que la mobilité de l'enseignant dans la classe (Martin, 2002). Ce travail bibliographique nous a permis d'évoquer des pistes de recommandations du type «l'organisation en îlots faciliterait l'intégration des technologies de réalité virtuelle par la création de classes d'apprentissage actif ».

15 En complément de ce travail, nous avons ensuite mis en œuvre des focus group impliquant des enseignants et chefs de travaux de lycées de plusieurs académies. L'objectif était d'analyser l'existant et de fournir des préconisations en termes d'intégration physique et pédagogique de la plate-forme dans les salles de classe. À l'issue de ces ateliers, nous avons collecté des données sur les dispositions actuelles des salles d'activités pratiques (par exemple, dimensions, encombrements matériels et configuration), les activités pédagogiques actuelles (par exemple, activités pratiques tournantes au rythme de deux séances de deux heures par semaine, réparties sur trois 
semaines), les activités pédagogiques envisagées avec les dispositifs de réalité virtuelle (par exemple, mettre un groupe d'élèves sur l'application venturi à un moment donné pendant qu'un autre groupe travaille sur un chauffe-eau solaire) ainsi que sur les dispositions futures des salles intégrant ces équipements sous forme de plan (Figure 2).

Fig. 2. Exemple de plan de salles de classe sur lequel les emplacements envisagés d'un V/Screen et d'une V/Station sont positionnés issus d'un focus group

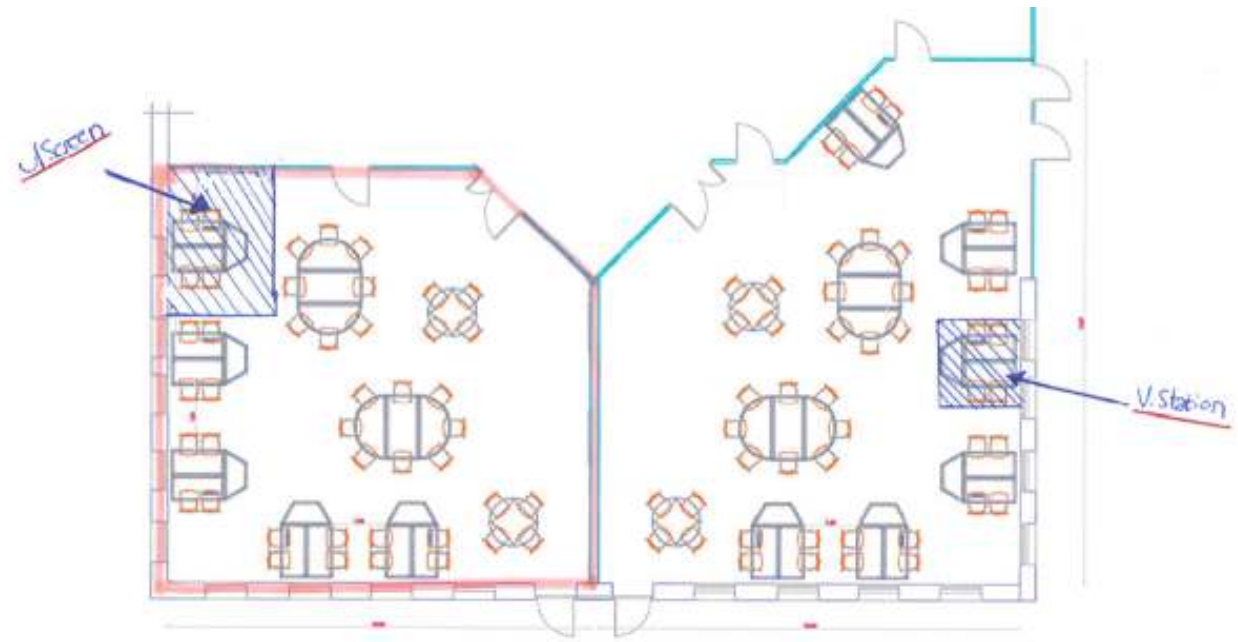

La littérature nous a permis d'aboutir à des préconisations générales pour l'organisation des salles de classe comportant des dispositifs de réalité virtuelle. En parallèle, les focus group nous ont permis de proposer des spécifications précises fondées sur les usages actuels (par exemple, compréhension d'une théorie à partir d'exercices pratiques effectués sur différents systèmes allant des logiciels de simulation jusqu'à des dispositifs réels) et sur l'anticipation d'usages futurs envisagés avec les nouvelles technologies (par exemple, utilisation d'une application pour permettre de faire travailler les élèves en difficulté dans le cadre de la remédiation). En effet, la production d'idées résultant de telles activités de groupe est tout à fait pertinente dans le domaine de l'innovation (Bruseberg, McDonagh-Philip, 2002).

\section{Analyser les exigences des usagers}

\section{Co-construction de scénarios pédagogiques intégrant les futures TICE}

Les caractéristiques et les besoins des enseignants et des élèves ont ensuite été spécifiés au moyen de la formalisation de scénarios pédagogiques. Pour définir les contenus pédagogiques à implémenter dans nos applications, nous avons conduit des brainstormings auprès de soixante-cinq participants. Le brainstorming est un outil de créativité libre et ordonnée qui permet de rechercher en groupe et en toute liberté un maximum d'idées sur un thème donné ou d'inventer des solutions pour résoudre un problème (Baccino, Colombi, 2005). Ces participants étaient des chefs de travaux, des enseignants du baccalauréat technologique STI2D (Tronc Commun et spécialités «Énergie et Environnement ", "Architecture et Construction » et "Innovation technologique et Éco-conception »), de baccalauréats professionnels (c'est-à-dire, Électrotechnique, Conception Mécanique, Construction) et de BTS (c'est-à-dire, Conception de Produits Industriels, Conception et Réalisation de Systèmes Automatiques, Génie Civil, Industrialisation des Produits Mécaniques). Ils provenaient des trois académies. À l'issue 
des séances de brainstorming, nous avons sélectionné six concepts pédagogiques susceptibles d'être abordés lors d'activités pratiques au moyen d'une application logicielle (Tableau 2). Cette sélection a été effectuée sur la base du contenu pédagogique et de la pertinence d'utilisation de la réalité virtuelle. Chacun d'entre eux a donné lieu à des preuves de concepts qui ont été implémentées. Les preuves de concepts sont des prototypes développés rapidement et qui ne seront pas systématiquement utilisés dans la suite du projet. Ils sont apparentés aux prototypes basse-fidélité ou aux prototypes jetables dans les sciences de la conception.

Tableau 2. Concepts pédagogiques (colonne de droite) abordés dans le cadre de Travaux Pratiques (colonne du milieu) au moyen de Preuves de Concepts (colonne de gauche)

\begin{tabular}{|l|l|l|}
\hline $\begin{array}{l}\text { Preuves de } \\
\text { Concepts }\end{array}$ & Objectifs des TP des & Concepts pédagogiques \\
\hline $\begin{array}{l}\text { Écoulement de } \\
\text { fluide }\end{array}$ & $\begin{array}{l}\text { Appréhender } \\
\text { phénomènes liés à l'effet } \\
\text { Venturi }\end{array}$ & $\begin{array}{l}\text { Conservation du débit, conservation de l'énergie, } \\
\text { réversibilité de la pression dynamique }\end{array}$ \\
\hline $\begin{array}{l}\text { Confort } \\
\text { acoustique }\end{array}$ & $\begin{array}{l}\text { Appréhender la notion de } \\
\text { confort acoustique }\end{array}$ & $\begin{array}{l}\text { Perception du son en fonction des revêtements des } \\
\text { parois, occultations, réverbération }\end{array}$ \\
\hline $\begin{array}{l}\text { Moteur } \\
\text { thermique }\end{array}$ & $\begin{array}{l}\text { Appréhender la notion de } \\
\text { chaine d'énergie }\end{array}$ & $\begin{array}{l}\text { Montage d'un moteur, rôles des différents organes, } \\
\text { séquencement des 4 temps, transformation d'un } \\
\text { mouvement linéaire en un mouvement de rotation }\end{array}$ \\
\hline $\begin{array}{l}\text { Schéma } \\
\text { cinématique }\end{array}$ & $\begin{array}{l}\text { Concevoir et comprendre } \\
\text { cinématique modélisation }\end{array}$ & $\begin{array}{l}\text { Conception d'un schéma cinématique, liaisons } \\
\text { cinématiques, simulation cinématique }\end{array}$ \\
\hline $\begin{array}{l}\text { Gamme } \\
\text { montage }\end{array}$ & $\begin{array}{l}\text { Définir et valider une } \\
\text { gamme d'assemblage }\end{array}$ & $\begin{array}{l}\text { Réalisation d'une gamme de } \\
\text { compréhension des étapes-clés } \\
\text { bâtiment montage, }\end{array}$ \\
\hline
\end{tabular}

\section{Recommandations pour l'ergonomie du dispositif matériel de réalité virtuelle}

La conception de TICE émergentes nécessite de penser au positionnement physique des dispositifs d'interaction et de visualisation au-delà des aspects logiciels afin d'optimiser l'utilisabilité globale du système. Aussi, nous avons dû fournir aux concepteurs des spécifications techniques concernant le principal dispositif de réalité virtuelle à implémenter dans les lycées, la V/Station. Ce dispositif se compose d'un zSpace (écran 3D incliné de 27 pouces, système de suivi, stylet, lunettes stéréoscopiques passives) et d'un bras à retour d'effort (Figure 3).

Pour limiter les contraintes physiques liées à l'usage de la V/Station, nous avons adapté les normes NF-X-35-104 («postures et dimensions de l'homme au travail sur machines et appareils ) et NF-X-35-121 («travail sur écran de visualisation et clavier ») fondées sur les 
caractéristiques anthropométriques humaines. Ces normes nous ont permis de positionner les périphériques d'interaction sur la V/Station (bras à retour d'effort, par exemple) ainsi que les dispositifs de visualisation et de fournir des recommandations sur le mobilier.

Fig. 3. V/Station @CLARTE

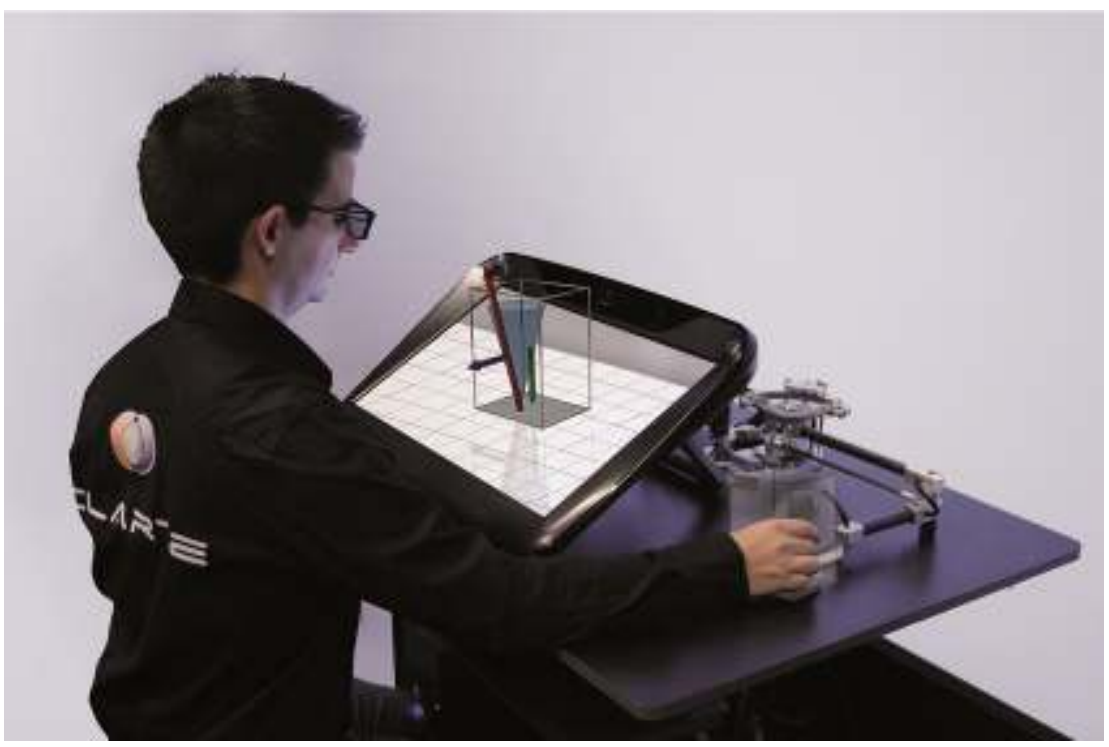

\section{Évaluer les solutions développées}

\section{Évaluation de l'utilité et l'utilisabilité perçues, de l'expérience-utilisateur et de l'acceptabilité des preuves de concepts}

L'évaluation des preuves de concepts précédemment évoquées (Tableau 2) a impliqué deux chefs de travaux et quatorze enseignants de BTS, de Bac Scientifique spécialité Sciences de l'Ingénieur et de la filière STI2D provenant des trois lycées de l'Académie de Nantes, de deux établissements de l'Académie de Créteil et d'un lycée de l'Académie de Rennes, ainsi que dix élèves de la filière STI2D d'un établissement de l'Académie de Nantes.

Chaque participant devait réaliser un test utilisateur. Un test utilisateur est une méthode utilisée pour évaluer des dispositifs interactifs avec des utilisateurs finaux à travers une mise en situation artificielle visant à étudier les comportements des utilisateurs face à un dispositif matériel et/ou logiciel (Baccino, Colombi, op. cit.). Elle présente l'avantage de permettre une confrontation de l'outil en cours de conception aux utilisateurs (contrairement aux méthodes d'inspection ergonomique n'impliquant pas les utilisateurs finaux) et de mesurer les opinions, les attitudes et les performances de ces derniers avec l'outil.

Concrètement, l'utilisateur devait compléter un questionnaire signalétique (c'est-à-dire, date de naissance, sexe, lycée etc.), réaliser un ensemble de tâches explicitées dans une fiche-consigne puis un questionnaire post-passation.

Ce dernier questionnaire nous a permis de collecter des données sur l'expérience vécue (c'est-à-dire, utilité et utilisabilité perçues, expérience utilisateur, acceptabilité) ainsi que les points positifs, les points à améliorer et les suggestions d'amélioration en termes 
d'usages (par exemple, cohérence avec les démarches pédagogiques d'investigation), de propriétés (par exemple, trop de dispositifs matériels d'interaction : clavier, terminal mobile et bras à retour d'effort) et de fonctionnalités (par exemple, pouvoir faire varier la géométrie du tube de Venturi).

\section{Évaluation de l'utilisabilité des prototypes}

Des prototypes, c'est-à-dire des applications nécessitant de légers développements supplémentaires pour être finalisés) ont ensuite été implémentés (Tableau 3).

Tableau 3. Prototypes (colonne de droite) et objectifs pédagogiques associés (colonne de gauche)

\begin{tabular}{|l|l|}
\hline Prototypes & Objectifs pédagogiques \\
\hline Venturi & $\begin{array}{l}\text { Appréhender les notions de conservation de l'énergie et de réversibilité } \\
\text { de la pression dynamique }\end{array}$ \\
\hline Vertige & Sensibiliser les élèves de filière professionnelle au travail en hauteur \\
\hline $\begin{array}{l}\text { Résistance } \\
\text { matériaux }\end{array}$ & $\begin{array}{l}\text { Approfondir le concept de résistance des matériaux au travers de la } \\
\text { flexion d'une poutre }\end{array}$ \\
\hline Éditeur cinématique & $\begin{array}{l}\text { Appréhender la notion de cinématique d'un mécanisme et de corréler la } \\
\text { représentation schématique avec l'assemblage de pièces mécaniques }\end{array}$ \\
\hline $\begin{array}{l}\text { Forces } \\
\text { frottements } \\
\text { statiques }\end{array}$ & dexpérimenter les notions d'équilibre d'un solide \\
\hline Virtualkart & $\begin{array}{l}\text { Comprendre la modélisation de la chaine d'énergie et d'appréhender la } \\
\text { transmission de puissance }\end{array}$ \\
\hline
\end{tabular}

Certaines preuves de concept ont été sélectionnées pour être améliorées en termes de fonctionnalités, de propriétés et de périphériques, en vue de devenir des prototypes (e.g. venturi, Figure 4). 
Fig. 4. Venturi, un tube de venturi modélisé faisant travailler les notions de conservation de l'énergie et de réversibilité de la pression dynamique CCLARTE

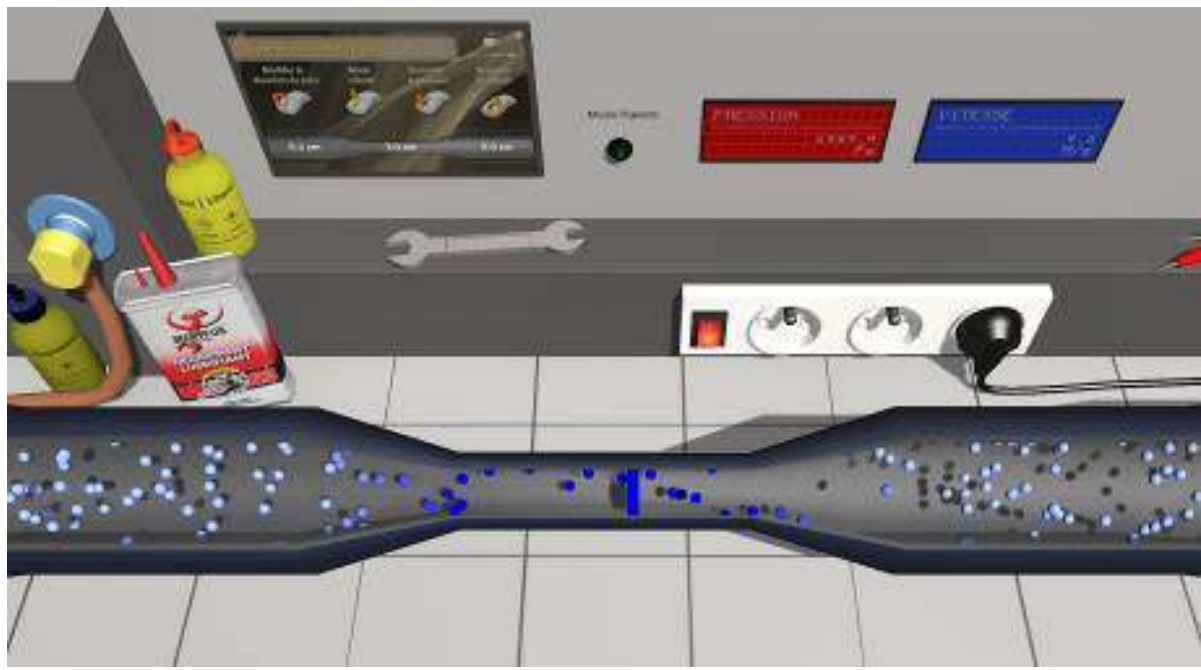

Les enseignants et les élèves ayant contribué aux tests ont discuté de l'utilité de certaines applications et suggéré de nouveaux contenus pédagogiques. Ainsi, certaines preuves de concepts ont été remplacées par de nouvelles applications (e.g. Wit4Pro a été remplacée par vertige, Figure 5).

Fig. 5. Vertige, un environnement virtuel visant à sensibiliser des élèves de filières professionnelles au travail en hauteur au moyen d'un oculus rift CCLARTE

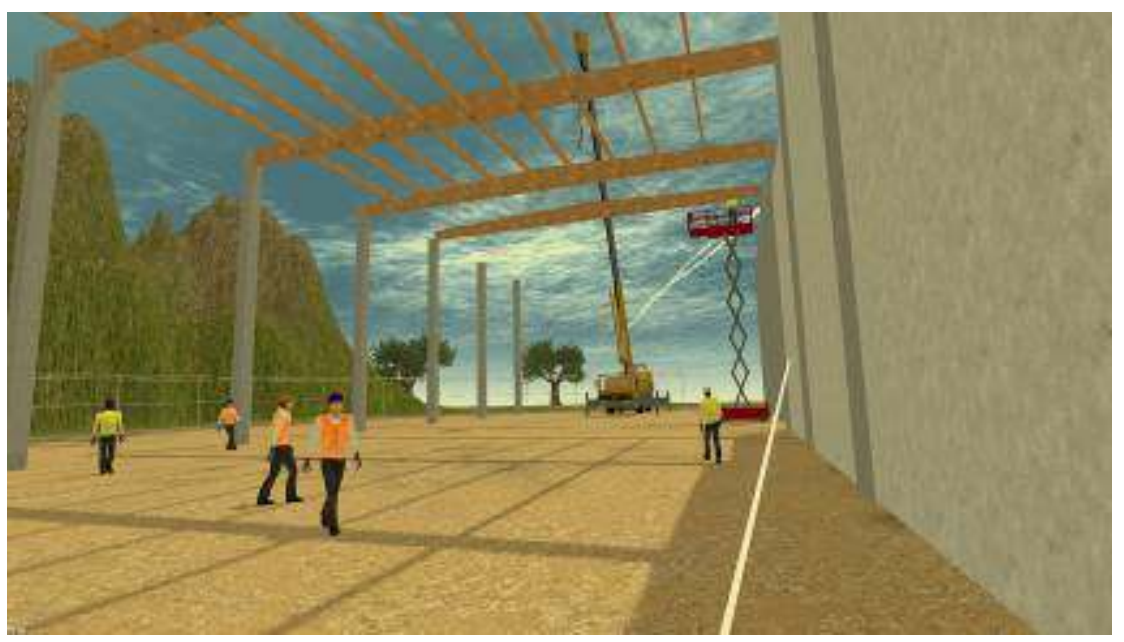

Ces nouveaux prototypes ont, de fait, dû faire l'objet de nouvelles évaluations en cours de projet afin de proposer aux lycées des applications finales optimisées spécifiquement en termes d'utilisabilité. Les prototypes évalués étaient : venturi (Figure 4), vertige (Figure 5), résistance des matériaux (un banc d'essai virtuel permettant d'approfondir le concept de résistance des matériaux au travers de la flexion d'une poutre), éditeur cinématique (application permettant d'appréhender la notion de cinématique d'un mécanisme et de corréler la représentation schématique avec l'assemblage de pièces mécaniques), forces de frottements statiques (environnement permettant d'expérimenter les notions d'équilibre d'un solide) et virtualkart (un kart virtuel permettant aux élèves de mieux comprendre la modélisation de la chaine d'énergie et l'appréhension de la transmission de puissance). 
28 En complément des méthodes d'évaluation impliquant les utilisateurs finaux, une méthode particulièrement rapide à mettre en œuvre pour améliorer l'utilisabilité des dispositifs interactifs est l'évaluation heuristique. Les évaluations heuristiques permettent de déterminer le nombre d'attributs d'un dispositif informatique entrant en adéquation avec des règles d'utilisabilité. Elles permettent de détecter les points positifs et les points à améliorer, et ainsi en déduire des recommandations (Baccino, Colombi, op. cit.).

29 Ces évaluations ont été réalisées par deux experts en ergonomie informatique. Après avoir repéré les problèmes d'utilisabilité, ces experts ont suggéré des pistes d'amélioration (Bach, 2004). Au total, 90 recommandations ont été proposées, il s'agissait de suggestions relatives à l'interaction homme-machine (par exemple, dans l'application "résistance des matériaux», le choix d'un matériau doit entrainer un changement d'apparence de la poutre) et de préconisations spécifiques aux environnements informatiques pour l'apprentissage humain (par exemple, insérer une synthèse explicative sur les phénomènes physiques explorés).

\section{Évaluation de l'appropriation des solutions finalisées implantées dans les lycées}

30 Au-delà de cette évaluation visant à améliorer des prototypes pour optimiser les TICE finales, des évaluations dans les lycées visant à mesurer l'appropriation de ces dernières par les enseignants et les élèves à travers un retour d'expérience ont ensuite été réalisées. En effet, dans chaque lycée partenaire, chaque application a été utilisée dans le cadre d'une activité pédagogique réelle (c'est-à-dire, activités pratiques, cours, remédiation) impliquant un enseignant et un groupe d'élèves. Ces situations d'utilisation sont écologiques dans la mesure où elles n'ont pas été programmées avec la seule finalité d'une évaluation, mais bien parce que l'utilisation d'une application spécifique (par exemple, venturi) s'intègre dans le programme scolaire au moment de l'année où l'enseignant aborde une thématique spécifique (par exemple, écoulement de fluide). Ainsi, toutes les applications n'ont pas pu être intégrées à ce retour d'expérience, et seules cinq applications ont été utilisées dans ce cadre: virtualkart, venturi, forces de frottements statiques et résistance des matériaux.

31 Ce retour d'expérience a été effectué au moyen d'entretiens semi-directifs ${ }^{7}$ auprès des enseignants dans la semaine qui a suivi l'utilisation de la plate-forme et de questionnaires que les enseignants ont fait passer aux élèves ayant utilisé l'application à la fin de la séance de cours.

$\mathrm{Au}$ total, neuf enseignants et 148 élèves provenant des trois lycées de l'Académie de Nantes ont participé.

Les entretiens avec les enseignants nous ont permis de collecter des données sur :

- La préparation de l'activité pédagogique intégrant la TICE (par exemple, la préparation de l'activité a nécessité au moins une dizaine d'heures pour préparer et tester plusieurs dispositifs en plus de l'application Virtualkart afin de pouvoir proposer aux binômes d'élèves plusieurs activités),

- L'organisation de la séance (par exemple, l'activité proposée intégrant virtualkart consistait à préparer un kart électrique pour une course. Les élèves étaient répartis en binômes et devaient mener à bien une des activités à réaliser : par exemple, un groupe était chargé du dimensionnement de la chaîne d'énergie tandis qu'un autre groupe devait mesurer la vitesse du kart avec un capteur), 
- L'implantation dans les salles de classe (par exemple, déplacement de la V/Station dans la classe afin d'avoir une vision circulaire sur tous les élèves).

Les données collectées grâce aux questionnaires ont montré que les TICE développées dans le projet favorisaient un très bon niveau d'acceptabilité (Venkatesh, Morris, Davis et al., op. cit.) et d'expérience-utilisateur (Hassenzahl, 2011). Par exemple, l'application venturi a été perçue comme utile (utilité perçue) ; les élèves qui l'ont utilisé souhaiteraient la réutiliser dans le cadre d'une autre activité pédagogique (intention d'usage). Elle a également été perçue comme innovante, originale, captivante et haut de gamme (c'est-àdire, qualités hédoniques), claire, simple, utilisable et fiable (c'est-à-dire, qualités pragmatiques) ainsi que plaisante, satisfaisante, enthousiasmante et bonne (c'est-à-dire, qualités affectives).

Tableau 4. Score pour l'application venturi (sur une échelle de Likert à 7 points)

\begin{tabular}{|l|c|l|l|l|l|}
\hline & \multicolumn{2}{|c|}{ Acceptabilité } & \multicolumn{2}{c|}{ Expérience-utilisateur } \\
\hline & $\begin{array}{l}\text { Utilité } \\
\text { perçue }\end{array}$ & $\begin{array}{l}\text { Intention } \\
\text { d'usage }\end{array}$ & $\begin{array}{l}\text { Qualités } \\
\text { pragmatiques }\end{array}$ & $\begin{array}{l}\text { Qualités } \\
\text { hédoniques }\end{array}$ & $\begin{array}{l}\text { Qualités } \\
\text { affectives }\end{array}$ \\
\hline Moyenne & 6,07 & 6,23 & 6,15 & 6,14 & 6,06 \\
\hline Écart-Type & 1,03 & 1,05 & 0,94 & 0,94 & 0,95 \\
\hline Valeur minimale & 2 & 2 & 3 & 3 & 3 \\
\hline Valeur maximale & 7 & 7 & 7 & 7 & 7 \\
\hline
\end{tabular}

Les élèves ont également évoqué certains aspects qui mériteraient d'être améliorés (par exemple, rajouter la perte de charge dans l'application venturi, la gâchette inférieure du bras à retour d'effort est difficile d'accès). D'autres suggestions concernant l'équipement de réalité virtuelle et le contenu pédagogique ont été proposées par les enseignants lors des entretiens (par exemple, remplacer le compteur analogique par le compteur numérique pour pouvoir effectuer des mesures précises dans l'application virtualkart).

\section{Conclusion}

La conception de TICE émergentes entraine de nombreuses itérations conception évaluation dans lesquelles il s'agit d'intégrer différentes populations d'utilisateurs finaux. Cela implique des coûts financiers et temporels importants relatifs non seulement aux améliorations informatiques comme dans les modèles du génie logiciel, mais aussi aux études expérimentales ou aux études de terrain réalisées. Cette implication des utilisateurs finaux est toutefois nécessaire pour développer un produit optimal en termes d'adéquation avec leurs besoins conscients ou latents. Dans le cadre du projet VirtualiTeach, impliquer les chefs de travaux, les enseignants et les élèves a eu plusieurs effets positifs, dans la mesure où cela a permis de 1) fournir aux concepteurs des informations sur les contextes d'usages dans lesquels les TICE seraient implantées, 2) établir des scénarios pédagogiques intégrant ces ressources numériques, 3) faire évoluer des prototypes en cours de conception en participant aux tests utilisateurs des preuves de 
concepts et 4) favoriser l'appropriation des applications finales dans les évaluations en les utilisant dans des situations pédagogiques écologiques. Elle a pour objectif d'adapter le mieux possible la ressource numérique aux besoins et aux pratiques de ceux qui en auront l'usage. Au-delà de ces contributions centrales, quelques précautions doivent être considérées pour une intégration réussie des utilisateurs finaux.

Une des spécificités de la conception des TICE est la variété et la diversité des utilisateurs potentiels. En effet, le besoin et la situation de formation future intégrant la technologie se construisent en référence à plusieurs populations spécifiques d'utilisateurs qui correspondent aux chefs de travaux, aux enseignants et aux élèves issus de onze lycées différents. Si cela présente l'avantage que les populations soient bien identifiées, les attentes et les caractéristiques de chaque population sont différentes. Pour autant, une sollicitation de tous les utilisateurs finaux n'est pas toujours nécessaire notamment dans les premières phases du projet. Une première précaution est alors de cibler les utilisateurs concernés et pertinents pour chaque étape du processus. Typiquement, les chefs de travaux et les enseignants sont les mieux placés pour nous décrire les contextes d'usage, la manière dont ils pensent intégrer physiquement et pédagogiquement les dispositifs dans les salles de classe et pour co-construire avec les concepteurs les scénarios pédagogiques. De la même manière, les élèves et les enseignants sont tout à fait pertinents pour nous faire un retour suite à des utilisations de prototypes haute-fidélité (en laboratoire ou en lycée) afin d'améliorer les fonctionnalités et les propriétés de ces TICE.

La seconde précaution est l'instauration d'un climat favorable entre les concepteurs et les utilisateurs. En effet, l'implication des utilisateurs finaux n'est pas toujours bien perçue par les concepteurs. La mise en place de réunions régulières au cours du développement peut favoriser le développement d'une confiance mutuelle entre concepteurs et utilisateurs permettant aux premiers de mieux comprendre les activités des enseignants et les attentes des élèves d'une part, et aux seconds de mieux cerner les potentialités et les verrous techniques des concepteurs d'autre part.

La troisième précaution, étroitement liée à la seconde, concerne l'articulation à trouver dans la conception et dans l'implication des utilisateurs entre l'existant et la projection d'une situation future probable. S'il est nécessaire de bien caractériser le contexte et l'activité existants (Boccara, Delgoulet, 2015) ainsi que les outils pédagogiques utilisés dans ces situations (par exemple, banc d'essai réel utilisé en STI2D), ce n'est pas suffisant pour permettre aux chefs de travaux et aux enseignants de se projeter dans une situation future potentielle dans laquelle ces dispositifs seraient implantés. Il est, en effet, nécessaire de compléter les méthodes d'analyse de l'activité classiquement utilisées avec des méthodes favorisant l'anticipation de ces nouveaux usages et situations. Par exemple, les preuves de concepts ont aidé les utilisateurs à identifier le potentiel de ces futurs outils et à élaborer puis à sélectionner les caractéristiques (c'est-à-dire, fonctionnalités, propriétés) à implémenter afin qu'ils soient pertinents pour les situations futures qu'ils imaginent (Loup-Escande, Dominjon, Perret et al., 2013). Dans cette perspective, les utilisateurs ne sont plus seulement une source d'informations sur l'activité existante, ils participent activement à la conception de ces situations futures probables au travers d'hypothèses sur les besoins, fonctionnalités, propriétés, usages et activités futurs possibles. Cette participation doit être facilitée ultérieurement à l'implantation de ces outils dans les situations de formation (c'est-à-dire, pendant le suivi) dans la mesure où seules les situations réelles de formation outillées permettront aux enseignants et aux 
élèves d'acquérir de nouvelles compétences sur la manière d'enseigner et d'apprendre avec ces technologies émergentes d'une part, et de conforter (ou pas) les hypothèses préalables quant à ces situations. La conception centrée-utilisateur prend classiquement fin lorsque les solutions pédagogiques innovantes sont suffisamment finalisées pour être insérées dans leurs situations d'usage. En outre, si cette démarche de conception suggère un suivi ultérieurement à l'intégration du dispositif interactif dans une situation réelle afin d'identifier notamment les difficultés rencontrées par les utilisateurs, ce suivi est généralement réalisé sur une courte période pour permettre aux concepteurs d'implémenter les modifications suggérées (Bastien, Scapin, 2004). La conception centréeutilisateur n'a pas pour objectif d'analyser l'évolution à moyen et à long terme (c'est-àdire, sur plusieurs mois voire plusieurs années) des besoins, des caractéristiques des utilisateurs (c'est-à-dire, enseignants et élèves) et de leurs pratiques au fur et à mesure de l'usage de ces technologies. Cet objectif est plutôt l'ambition des approches de conception participative et continuée au travers de l'usage (Rabardel, Beguin, 2005 ; Loup-Escande, 2010) qui sont tout à fait pertinentes pour considérer ces évolutions inhérentes à toute innovation (Brangier, 2015). La quatrième précaution est, par conséquent, d'éviter l'arrêt complet des évaluations centrées utilisateurs. En effet, ce type de dispositif sera amené à évoluer en fonction des contenus ou des technologies de réalité virtuelle. Il est donc essentiel de maintenir des évaluations avec les utilisateurs en vue d'affiner les usages, les fonctions et l'efficacité du dispositif. Cela permettrait de répondre à de nombreuses questions de recherche, comme : comment disposer les îlots dans la classe? De combien d'élèves sont composés les îlots? Quel type d'activité est propice à un travail collaboratif par îlot avec la réalité virtuelle?, Est-ce-que la motivation des élèves est maintenue dans le temps ?, etc.

\section{BIBLIOGRAPHIE}

Ausburn, L. J., Ausburn, F.B. (2004). Desktop Virtual Reality: A Powerful New Technology for Teaching and Research in Industrial Teacher Education. Journal of Industrial Teacher Education, 41 (4), pp. 33-58.

Baccino, T., Colombi, T. (2005). Mesure de l'utilisabilité des interfaces, Paris : Hermès Science Publisher.

Bach, C. (2004). Élaboration et validation de Critères Ergonomiques pour les Interactions HommeEnvironnements Virtuels. Thèse de Doctorat. Université de Metz.

Baker, P. M. (1984). Seeing is behaving: Visibility and participation in small groups. Environment and Behavior, 16(2), pp. 159-184.

Bastien, J. M. C., Scapin, D. (2004). La conception de logiciels interactifs centrée sur

l'utilisateur : étapes et méthodes. In P. Falzon (Ed.), Ergonomie. PUF, Paris.

Brangier, E. (2015). Innover avec l'ergonomie, Constructif, 41, pp. 19-21. 
Boccara, V., Delgoulet, C. (2015) Une démarche d'analyse du travail pour la conception d'un outil virtuel de formation. Activités, 12(2).

Bruseberg, A., McDonagh-Philip, D. (2002). Focus groups to support the industrial/product designer: a review based on current literature and designers' feedback. Applied Ergonomics, 33(1), pp. 27-38.

Burkhardt, J.-M., Lourdeaux, D., Mellet-d'Huart, D. (2003) La conception des environnements virtuels pour l'apprentissage". In Fuchs P., Moreau G. Eds. Le traité de la réalité virtuelle. Tome 2, 2 ème édition. Presse de l'école des Mines de Paris.

Codd, A. M., Choudhury, B. (2011). Virtual reality anatomy: Is it comparable with traditional methods in the teaching of human forearm musculoskeletal anatomy?. Anatomical Sciences Education, 4(3), pp. 119-125.

Crétin-Pirolli, R., Cottier, P. (2009). Conception et appropriation d'un outil TICE particulier : prise en compte des facteurs institutionnels. Actes EPAL.

Dede, C. (2009). Immersive interfaces for engagement and learning. Science, 323(5910), pp. 66-69.

Fleury, S., Jamet, E., Loup-Escande, E., Ghorbel, A., Lemaitre, A., Anquetil, E. (2013). Towards Specifications for Automatic Recognition Software: An Example of a User-Centred Design. Journal of Software Engineering and Applications, 6(10A), pp. 1-4.

Fisher, K. (2010). Environnements pédagogiques actifs facilités par la technologie : Une évaluation. CELE Échanges, Centre pour des environnements pédagogiques efficaces, No.2010/07, Éditions OCDE.

Freitas, S., Neumann, T. (2009). The use of 'exploratory learning' for supporting immersive learning in virtual environments. Computers \& Education, 52(2), pp. 343-352.

Ganier, F., Hoareau, C., Devillers, F. (2013). Évaluation de l'apprentissage de procédures avec GVT, environnement virtuel d'apprentissage d'opérations de maintenance. Le Travail Humain.

Hassenzahl, M. (2011) The effect of perceived hedonic quality on product appealingness, International Journal of Human-Computer Interaction, 13, pp. 481-499.

Hew, K. F., Cheung, W. S. (2010). Use of three-dimensional (3-D) immersive virtual worlds in K-12 and higher education settings: a review of the research. British Journal of Educational technology, 41 (1), pp. 33-55.

Hix D., Swan, J. E., Gabbard, J. L., McGee, M., Durbin, J., King, T. (1999). User-Centered Design and Evaluation of a Real-Time Battlefield Visualization Virtual Environment. IEEE Virtual Reality, pp. 96-103.

Jamet, E., Trémenbert, J., Deguillard, G., Erhel S. (2011). Un exemple de méthodologie de conception centrée sur l'utilisateur de produits innovants : le projet duoTV. In G. Dang Nguyen \& P. Créach (Eds.) Recherches sur la Société du Numérique et ses Usages, L'Harmattan.

Jou, M., Wang, J. (2013). Investigation of effects of virtual reality environments on learning performance of technical skills. Computers in Human Behavior, 29(2), 433-438. doi: http:// dx.doi.org/10.1016/j.chb.2012.04.020

Loup-Escande, E. (2010). Vers une conception centrée sur l'utilité : une analyse de la co-construction participative et continue des besoins dans le contexte des technologies émergentes. Thèse de Doctorat. Université d'Angers.

Loup-Escande, E., Burkhardt J.-M., Richir, S. (2013). Anticiper et évaluer l'utilité dans la conception ergonomique des technologies émergentes, Le Travail Humain, 76(1), pp. 25-55. 
Loup-Escande, E., Dominjon, L., Perret, D., Erhel, S., Jamet, E., Michinov, N., Andriot, C., Gravez, P., Ragot, M. (2013). La démarche de Conception Centrée-Utilisateur en Réalité Virtuelle : l'exemple du projet VirtualiTeach. AFRV 2013.

Maguire, M. (2001). Methods to support human-centred design, International Journal of HumanComputer Studies, 55(4), pp. 587-634.

Martin, S. H. (2002). The classroom environment and its effects on the practice of teachers. Journal of Environmental Psychology, 22(1-2), pp. 139-156.

Mikropoulos, T. A., Natsis, A. (2011). Educational virtual environments: A ten-year review of empirical research (1999-2009). Computers \& Education, 56(3), pp. 769-780.

Plantard, P. (1999). De l'éducation virtuelle. Ville École Intégration, 119, Ed. CNDP, Paris, pp. 142-156.

Rabardel, P., Beguin, P. (2005). Instrument mediated activity: from subject development to anthropocentric design. Theoretical Issues in Ergonomic Science, 6(5), pp. 429-461.

Venkatesh, V., Morris, M. G., Davis, G. B., Davis, F. D. (2003), User acceptance of information technology: Toward a unified view, MIS QUART, 27(3), pp. 425-478.

Virvou, M., Katsionis, G., Manos, K. (2005). Combining software games with education: evaluation of its educational effectiveness. Educational Technology and Society, 8(2), pp. 54-65.

Whiteside, A., Brooks, D.C., Walker, J.D. (2010). Making the case for space: three years of empirical research on formal and informal learning environments. Educause Quarterly, 33(3).

Witmer, B. G., Singer, M. J. (1998). Measuring Presence in Virtual Environments: A Presence Questionnaire, Presence, 7(3), pp. 225-240.

\section{NOTES}

1. Les chefs de travaux sont également appelés «directeurs délégués aux enseignements technologiques et professionnels ». Ce sont généralement d'anciens enseignants certifiés ou agrégés ayant exercés dans des établissements techniques et professionnels.

2. Il s'agit d'un projet de l'appel à projets $n^{\circ} 2$ : «Services Numériques Innovants Pour L'eEducation", financé par la caisse des dépôts et des consignations dans le cadre des investissements d'avenir, la région Pays de la Loire et la région Bretagne.

3. STI2D : Sciences et Technologies de l'Industrie et du développement Durable.

4. Académie de Nantes : Lycée Réaumur, Lycée Saint-Joseph de la Joliverie, Lycée Aristide Briand, Lycée Brossaud-Blancho.

5. Académie de Créteil : Lycée Louis Armand, Lycée Gustave Eiffel, Lycée Léonard de Vinci, Lycée François Mansart.

6. Académie de Rennes : Lycée Mendès France, Lycée Bréquigny, Lycée Joliot-Curie.

7. Les entretiens semi-directifs consistent à aborder un certain nombre de thèmes au moyen de questions-relances contenues dans un guide d'entretien. 


\section{RÉSUMÉS}

La conception d'Environnements Virtuels Éducatifs (EVE) efficaces nécessite l'implication des utilisateurs finaux. Aussi, nous avons mis en place un processus de conception centrée-utilisateur (norme IS0 9241-210) dans le cadre d'un projet visant la conception d'un environnement virtuel favorisant l'apprentissage de concepts scientifiques (projet VirtualiTeach). L'objectif de cet article est de formaliser ce qu'est une conception avec et pour les utilisateurs finaux dans le contexte des EVE visant à favoriser l'apprentissage de concepts scientifiques et d'en identifier les apports. Pour ce faire, nous décrivons les contributions effectives de plusieurs profils d'utilisateurs (par exemple, les enseignants, les élèves) à la compréhension et à la spécification des contextes d'usage, à l'identification des besoins des utilisateurs et aux évaluations de solutions intermédiaires et finales.

Designing effective Educational Virtual Environments (EVE) requires the involvement of end users. To do so, we have implemented a user-centered design process (IS0 9241-210) in a project to design a virtual learning environment of scientific concepts (VirtualiTeach project). The main purpose of this article is to formalize a user-centered design process in the context of EVE for learning scientific concepts and to identify the contributions. In the first two phases (context analysis and user requirement analysis), the project's various stakeholders contributed to the environment design process through workshops and interviews. In the third phase (usercentered evaluation), user tests were conducted to assess design solutions (for example, mockup, prototypes, final applications).

\section{INDEX}

Mots-clés : TICE, EVE, conception centrée-utilisateur, réalité virtuelle, évaluation

Keywords : ICTE, EVE, user-centered design, virtual reality, evaluation

\section{AUTEURS}

\section{ÉMILIE LOUP-ESCANDE}

CRP-CPO EA7273, Université de Picardie Jules Verne, Amiens, France emilie.loup-escande@u-picardie.fr

\section{ÉRIC JAMET}

Centre de Recherches en Psychologie, Cognition et Communication, Université Rennes 2, Rennes, France

eric.jamet@uhb.fr 


\section{MARTIN RAGOT}

Centre de Recherches en Psychologie, Cognition et Communication, Université Rennes 2, Rennes, France

martin.ragot@uhb.fr

\section{SÉVERINE ERHEL}

Centre de Recherches en Psychologie, Cognition et Communication, Université Rennes 2, Rennes, France

severine.erhel@uhb.fr

\section{NICOLAS MICHINOV}

Centre de Recherches en Psychologie, Cognition et Communication, Université Rennes 2, Rennes, France

nicolas.michinov@uhb.fr

\section{CLÉMENT PELTIER}

CLARTE, plate-forme de réalité virtuelle, Laval, France clement.peltier@clarte.asso.fr

\section{THOMAS LOPEZ}

CLARTE, plate-forme de réalité virtuelle, Laval, France

lopez@clarte.asso.fr 